Acad. Sci. U. S. A. 98:8850-8855.

3. Bard, F., et al. 2000. Peripherally administered antibodies against amyloid $\beta$-peptide enter the central nervous system and reduce pathology in a mouse model of Alzheimer disease. Nat. Med. 6:916-919.

4. Monsonego, A., et al. 2003. Increased T cell reactivity to amyloid $\beta$ protein in older humans and patients with Alzheimer disease. J. Clin. Invest. 112:415-422. doi:10.1172/JCI200318104.

5. Eriksen, J.L., et al. 2003. NSAIDs and enantiomers of flurbiprofen target $\gamma$-secretase and lower A 342 in vivo. J. Clin. Invest. 112:440-449. doi:10.1172/JCI200318162.
6. Morris, J.C., and Price, A.L. 2001. Pathologic correlates of nondemented aging, mild cognitive impairment, and early-stage Alzheimer's disease. J. Mol. Neurosci. 17:101-118.

7. Morgan, D., et al. 2000. A beta peptide vaccination prevents memory loss in an animal model of Alzheimer's disease. Nature. 408:982-985.

8. Janus, C., et al. 2000. A beta peptide immunization reduces behavioural impairment and plaques in a model of Alzheimer's disease. Nature. 408:979-982.

9. Dodart, J.C., et al. 2002. Immunization reverses memory deficits without reducing brain Abeta burden in Alzheimer's disease model
Nat. Neurosci 5:452-457.

10. Kotilinek, L.A., et al. 2002. Reversible memory loss in a mouse transgenic model of Alzheimer's disease. J. Neurosci. 22:6331-6335.

11. Nicoll, J.A., et al. 2003. Neuropathology of human Alzheimer disease after immunization with amyloid- $\beta$ peptide: a case report. Nat. Med. 9:448-452

12. Town, T., et al. 2001. Characterization of murine immunoglobulin $\mathrm{G}$ antibodies against human amyloid- $\beta 1-42$. Neurosci. Lett. 307:101-104.

13. Hock, C., et al. 2002. Generation of antibodies specific for $\beta$-amyloid by vaccination of patients with Alzheimer disease. Nat. Med. 8:1270-1275

\title{
Endocannabinoids and the regulation of body fat: the smoke is clearing
}

\section{Tamas L. Horvath \\ Department of Obstetrics and Gynecology, and Department of Neurobiology, Yale University School of Medicine, New Haven, Connecticut, USA}

\begin{abstract}
Endocannabinoids, endogenous ligands of cannabinoid receptor type 1 (CB1), have emerged as novel and important regulators of energy homeostasis. A report in this issue (see the related article beginning on page 423) demonstrates reduced body weight, fat mass, and appetite in $\mathrm{CB} 1^{-/-}$ mice. Examination of the underlying mechanisms reveals a dual role for endocannabinoids as they affect both appetite and peripheral lipolysis.
\end{abstract}

J. Clin. Invest. 112:323-326 (2003). doi:10.1172/JCI200319376

Chronically elevated energy expenditure without a corresponding increase in energy intake leads to wasting and death. Almost all species in the wild, and the great majority of the human race, struggle with such negative energy balance in their daily battle for survival. In sharp contrast, however, modern industrialized societies are threatened by the exact opposite: chronically increased energy intake without a respective increase in energy expenditure. This constellation leads to obesity and diabetes as well as a variety of life-threatening consequences of such diseases, such as can-

\footnotetext{
Address correspondence to: Tamas L. Horvath, Department of Obstetrics and Gynecology, and Department of Neurobiology, Yale University School of Medicine, 333 Cedar Street, FMB 339 New Haven, Connecticut 06520, USA. Phone: (203) 785-4597; Fax: (203) 785-4747; E-mail: tamas.horvath@yale.edu.

Conflict of interest: The author has declared that no conflict of interest exists.

Nonstandard abbreviations used: cannabinoid receptor type 1 (CB1); corticotropinreleasing hormone $(\mathrm{CRH})$; cocaineamphetamine-regulated transcript (CART) melanin-concentrating hormone (MCH).
}

cer and cardiovascular diseases $(1,2)$. While it appears intuitively obvious that in the majority of cases, positive energy balance should be corrected by changes in lifestyle and/or diet, the impressive dynamics of the spreading obesity epidemic (3) certainly suggests that, in modern industrialized civilizations, an efficient and safe pharmacological approach to treat obesity would be useful. In light of this, it is not surprising that within the past decades, increasing attention has been paid to central and peripheral regulatory components of energy metabolism to develop pharmacological modulators of appetite and energy expenditure. From this perspective, one of the great, and surprising, discoveries of the past decade was the revelation of an endocannabinoid system and its influences on appetite and metabolism (4-8).

Cannabinoids and endocannabinoids act via $G$ protein-coupled receptors. The strongest effects of endocannabinoids on behavior, including those related to food intake, appear to be mediated by cannabi- noid receptor type 1 (CB1), which is the predominant receptor type in the CNS. The molecular cascade triggered by CB1 activation has been studied in depth (reviewed in ref. 8). In short, the dominant $G$ protein subtypes activated by CB1 belong to the $\mathrm{G}_{\mathrm{i} / \mathrm{o}}$ family; these in turn alter electric properties of membranes, secondmessenger systems, and immediateearly genes. CB1 activation inhibits voltage-gated $\mathrm{L}, \mathrm{N}$, and $\mathrm{P} / \mathrm{Q} \mathrm{Ca}^{2+}$ currents, while activating $K^{+}$currents; and while agonists of CB1 induce the inhibition of adenylyl cyclase, they also induce the activation of focal adhesion kinase and MAPK. CB1associated activation of $\mathrm{G}$ proteins also underlies the stimulation of $\mathrm{NO}$ synthase. Current ideology suggests that the activation or inhibition of CB1 (mainly by fatty acid ethanolamides) influences the aforementioned subcellular events and results in changes in neurotransmitter release at the level of axon terminals (7). However, to date, the phenotype of neurons in the hypothalamic appetite center that are directly affected by cannabinoids has not been elucidated. In this issue of the JCI, a report by Cota et al. (9) greatly advances our understanding of this critical issue by pinpointing those neuropeptide systems in the hypothalamus that most likely mediate cannabinoid-induced changes in energy homeostasis.

\section{Cannabinoids and metabolism}

Anecdotal evidence regarding the robust effect of the recreational drug marijuana (Cannabis sativa) on appetite and food intake has been widely known for centuries (10). However, it was the discovery of marijuana's main psychoactive component, $\Delta^{9}$-tetra- 
hydrocannabinol, almost 40 years ago (11) that triggered prospective scientific endeavors to decipher the mechanism of action of this popular, albeit mainly illegal, herbal drug. Advances gained momentum when CB1 and CB2 and their endogenous ligands were discovered in the early 1990s (4-6). In the brain, only CB1 appears to be expressed (12) and mediates effects of endogenous cannabinoids (and most likely the psychoactive ingredients of marijuana) on synaptic transmission (7). It is intriguing to note that endocannabinoids act on presynaptic release of neurotransmitters (7) while apparently being produced postsynaptically (13). A synthetic receptor antagonist of $\mathrm{CB} 1$ was shown to powerfully reduce food intake and increase energy expenditure (14), and, subsequently endocannabinoids were shown to induce food intake (15). These observations paved the way for a possible drug candidate for treatment of obesity. In this issue of JCI, Cota et al. (9) report that CB1 gene disruption in mice (CB1-/mice) unmasked a dual role of endocannabinoids in the regulation of energy balance, including both central orexigenic and peripheral adipogenic effects. These studies, in conjunction with the fact that cannabinoid receptor antagonist compounds are currently in clinical trials as antiobesity drugs in Europe, raise the hope that single-drug therapies could be successful in the treatment of obesity.

\section{Central and peripheral mode of action of endocannabinoids}

The work by Cota et al. (9) and previous studies on cannabinoids will give rise to guarded optimism - yet again - that pharmacological strategies, now targeting the cannabinoid system, may succeed in counterbalancing overeating and a sedentary lifestyle. Previously, similar hopes were generated every time a novel molecule (hormone, peptide, or neurotransmitter) was discovered to affect energy metabolism. Cota et al. provide compelling evidence that endocannabinoids act at various regions of the hypothalamus that regulate energy homeostasis, including the lateral hypothalamus, arcuate nucleus, and paraventricular nucleus, in conjunction with direct effects in fat cells on lipogenesis to promote a positive energy balance. If the receptor, CB1, is blocked (in this case by knockout of the gene), the overall effect will be to decrease appetite and lipogenesis in white fat: $\mathrm{CB}^{-/-}$animals are leaner because their caloric intake is lower when they are young. However, in older animals, increased peripheral energy expenditure appears to be the predominant defense against adiposity in these animals compared with their wild-type littermates (9). Cota and colleagues show that CB1 is expressed in key hypothalamic peptidergic systems of appetite regulation, including those producing corticotropin-releasing hormone (CRH) in the paraventricular nucleus, cocaine amphetamine-

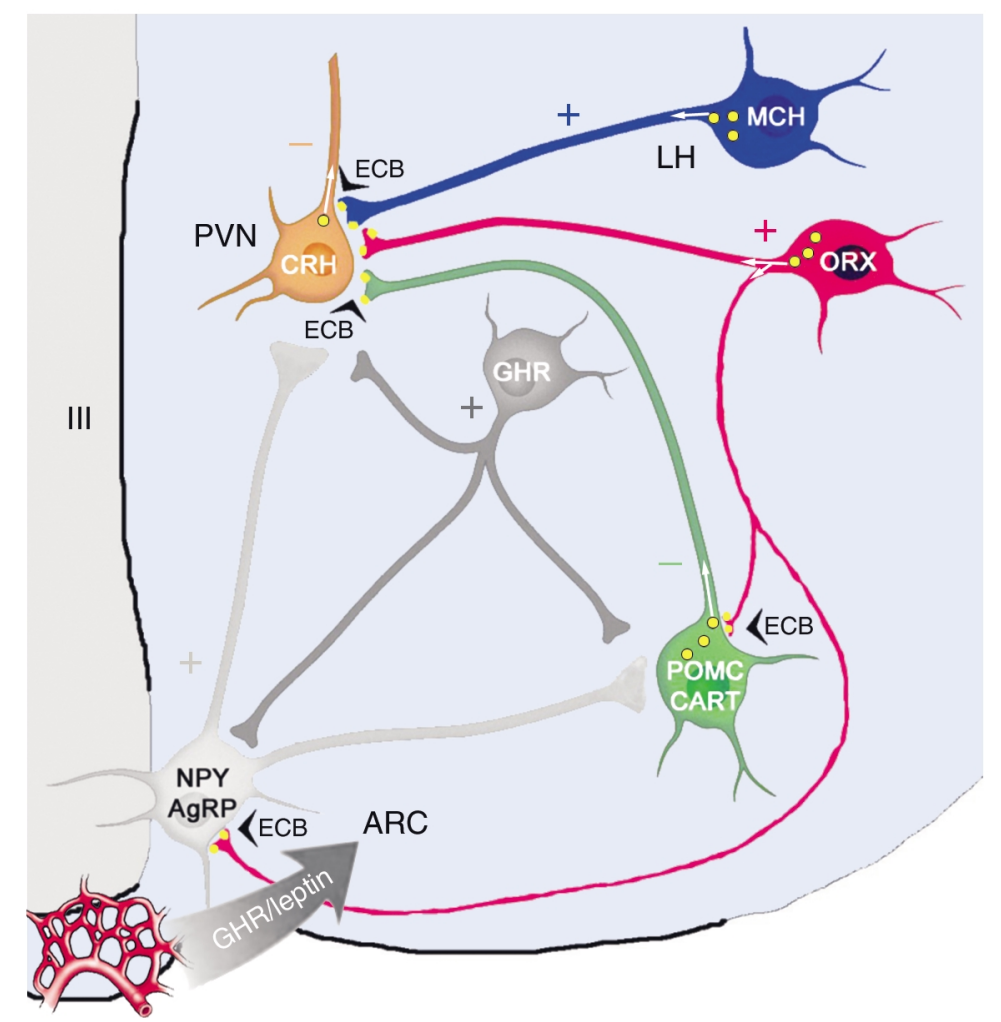

\section{Figure 1}

Schematic illustration showing the relationship between hypothalamic peptidergic circuits that express CB1 receptors (yellow cirlces), as described by Cota et al. (9), and some of those that do not. The former include cells that produce CART and pro-opiomelanocortin (POMC) in the arcuate nucleus (ARC); neurons that express $\mathrm{MCH}$ (blue) and orexin (ORX; red) in the lateral hypothalamic ( $\mathrm{LH})$ region; and neurons that produce $\mathrm{CRH}$ (brown) in the paraventricular nucleus (PVN). CB1 receptors are produced in the cytosol of these cells (yellow circles outlined in black) and are transported (white arrows) to axon terminals. Here, the receptors, upon activation (black arrowheads) by endocannabinoids (ECB) or other agonists, are thought to affect the release of neuromodulators $(\mathrm{MCH}, \mathrm{ORX}, \mathrm{CART}$, corticotropin-releasing factor) to the synaptic cleft. A population of neurons in the medial arcuate nucleus produces neuropeptide $Y$ (NPY) and agouti-related protein (AgRP), both of which are considered regulators of energy homeostasis. The NPY/AgRP-producing neurons, as well as those that produce ghrelin (GHR), were not found to contain CB1 receptors (9). GHR is also produced in the stomach and, together with the adipose hormone leptin, reaches hypothalamic peptidergic circuits via the circulation. The source of endocannabinoids in the hypothalamus is yet to be determined. Similarly, the hierarchical relationship between CB1-containing axon terminals and other axon terminals and their postsynaptic targets needs to be established to allow a better understanding of the hypothalamic signaling of cannabinoids. Putative inhibitory (-) and stimulatory $(+)$ influences on food intake of the various elements of the peptidergic system are shown. Note that this influence on food intake may not reflect electrophysiological effects of the respective peptides. III, third ventricle. 
regulated transcript (CART) in the arcuate nucleus, and melanin-concentrating hormone $(\mathrm{MCH})$ and orexin/hypocretin in the lateral hypothalamus-perifornical region. Therefore, it is likely that by modulating food intake and mechanisms regulating energy expenditure, endocannabinoids or their antagonists will affect the signaling flow between these hypothalamic circuits and other neuronal networks with which these peptidergic systems interact (Figure 1). This central mechanism may dominate cannabinoid effects at younger ages but appears to diminish with age (9). Whether it is chronological aging per se or other developmental processes, for example, puberty, that affects the function of relevant hypothalamic circuitry and thereby enables this impairment of cannabinoid signaling still needs to be clarified. In addition, it must be determined whether such a shift in endocannabinoid signaling during the aging process also occurs in humans. Nevertheless, the observation that $\mathrm{CB} 1$ is present in adipocytes and that CB1 activation affects lipogenesis provides an alternative and/ or complementary mechanism for the unidirectional effect of cannabinoids on energy balance.

\section{The endocannabinoid system as promising drug target for obesity} Cota and colleagues (9) have provided an insightful look into the mechanism of cannabinoid action. It is particularly intriguing that a system known to be an essential part of CNS mechanisms controlling reward and memory functions has now been identified as one of the very few orexigenic components of energy-balance regulation, in which targeted gene disruption is followed by a lean phenotype. Intriguingly, the other orexigenic system that demonstrates a similar phenotype after ablation is that which produces $\mathrm{MCH}$ (16), which Cota et al. now directly connect to the endogenous cannabinoid system. On the other hand, the arcuate nucleus neuropeptide $\mathrm{Y} /$ agouti-related protein (NPY/AgRP) system, which Cota et al. demonstrate is not directly targeted by endocannabinoid action, appears to be a less critical (or a functionally more redundant) player in the chronic maintenance of energy balance (17). However, disruption of NPY Y1 or Y2 receptors, which are intricately related to some of the peptidergic neurons $(18-21)$ that are shown by Cota et al. to express CB1 receptors, attenuate the development of type 2 diabetes $(22,23)$.

The hedonic component of hypercaloric nutrition (24) could possibly be targeted by a CB1 antagonist, which might be able to diminish the possibly addictive aspect of food intake in some individuals, in combination with decreasing orexigenic drive and lipogenesis. As depicted in Figure 1, there is a redundancy of connectivity among hypothalamic peptidergic systems, some of which simultaneously utilize other neurotransmitters and peptides as well (25). An intricate relationship exists between axonal processes and postsynaptic targets of the various systems, but interactions also occur between different axon terminals presynaptically. Thus, to understand endocannabinoid signaling within the hypothalamus, it will be necessary to combine classical light and electron microscopic analyses of chemically identified anatomical interactions with state-of-the-art electrophysiological approaches. Such approaches have recently been used to better understand the hypothalamic signaling of two other metabolic hormones, ghrelin and leptin (26, 27). Determining the central action of these molecules will most likely require the application of interdisciplinary techniques that include neurophysiology, molecular biology, biochemistry, and pharmacology. Regarding the cannabinoid system and obesity therapies, it will be particularly important to expand experimental studies to include other brain sites, including the cortex and basal forebrain, and their functions. The anecdotal and experimental evidence of marijuana's appetite-regulating effects also shows clear alterations of other CNS mechanisms, such as learning, memory, aggression, and addiction (10). While experimental design generally demands that one focus on only one type of behavior in the experimental system, this approach may have grave consequences for therapeutics. For example, the weight-reducing effects of an antagonist of CB1 could enhance attention, drive, and focus at the expense of emotions and social behavior. Nevertheless, as the smoke continues to clear, the cannabinoid system may prove to be an extremely promising target for the development of medications for obesity and cachexia (9).

\section{Acknowledgments}

The author's work on hypothalamic circuitry has been supported by NIH grants MH-59847, RR-14451, DK60711, and DK-51730.

1. Calle, E.E., Rodriguez, C., Walker-Thurmond, K., and Thun, M.J. 2003. Overweight, obesity, and mortality from cancer in a prospectively studied cohort of U.S. adults. N. Engl. J. Med. 348:1625-1638.

2. Eckel, R.H. 1997. Obesity and heart disease: a statement for healthcare professionals from the Nutrition Committee, American Heart Association. Circulation. 96:3248-3250.

3. Flegal, K.M., Carroll, M.D., Ogden, C.L., and Johnson, C.L. 2002. Prevalence and trends in obesity among US adults, 1999-2000. JAMA. 288:1723-1727.

4. Matsuda, L.A., Lolait, S.J., Brownstein, M.J., Young, A.C., and Bonner, T.I. 1990. Structure of a cannabinoid receptor and functional expression pf the cloned cDNA. Nature. 346:561-564.

5. Munro, S., Thomas, K.L., and Abu-Shaar, M. 1993. Molecular characterization of a peripheral receptor for cannabinoids. Nature. 365:61-65.

6. Devane, W.A., et al. 1992. Isolation and structure of a brain constituent that binds to the cannabinoid receptor. Science. 258:1946-1949.

7. Di Marzo, V., et al. 1998. Endocannabinoids: endogenous cannabinoid receptor ligands with neuromodulatory action. Trends Neurosci. 21:521-528

8. Reggio, P.H. 2002. Endocannabinoid structureactivity relationships for interaction at the cannabinoid receptors. Prostaglandins Leukot. Essent. Fatty Acids. 66:143-160.

9. Cota, D., et al. 2003. The endogenous cannabinoid system affects energy balance via central orexigenic drive and peripheral lipogenesis. J. Clin. Invest. 112:423-431. doi:10.1172/ JCI200317725

10. Scigliano, J.A. 1981. THC therapeutic research by independent and state-sponsored investigators: a historical review. J. Clin. Pharmacol. 21(Suppl. 8-9):113S-121S.

11. Gaoni, Y., and Mechoulam, R. 1964. Isolation, structure and partial synthesis of an active constituent of hashish. J. Am. Chem. Soc. 86:1646.

12. Marsicano, G., and Lutz, B. 1999. Expression of the cannabinoid receptor CB1 in distinct neuronal subpopulations in the adult mouse forebrain. Eur. J. Neurosci. 11:4213-4225.

13. Egertova, M., Giang, D.K., Cravatt, B.F., and Elphick, M.R. 1998. A new perspective on cannabinoid signalling: complementary localization of fatty acid amide hydrolase and the CB1 receptor in rat brain. Proc. R. Soc. Lond. B Biol. Sci. 265:2081-2085. 
14. Colombo, G., et al. 1998. Appetite suppression and weight loss after the cannabinoid antagonist SR 141716. Life Sci. 63:L113-L117.

15. Di Marzo, V., et al. 2001. Leptin-regulated endocannabinoids are involved in maintaining food intake. Nature. 410:822-825.

16. Shimada, M., Tritos, N.A., Lowell, B.B., Flier, J.S and Maratos-Flier, E. 1998. Mice lacking melanin-concentrating hormone are hypophagic and lean. Nature. 396:670-674.

17. Qian, S., et al. 2002. Neither agouti-related protein nor neuropeptide $\mathrm{Y}$ is critically required for the regulation of energy homeostasis in mice. Mol. Cell. Biol. 22:5027-5035.

18. Broberger, C., Landry, M., Wong, H., Walsh, J.N., and Hokfelt, T. 1997. Subtypes Y1 and Y2 of the neuropeptide $\mathrm{Y}$ receptor are respectively expressed in pro-opiomelanocortin- and neuropeptide-Y-containing neurons of the rat hypo- thalamic arcuate nucleus. Neuroendocrinology. 66:393-408

19. Broberger, C., Visser, T.J., Kuhar, M.J., and Hok felt, T. 1999. Neuropeptide Y innervation and neuropeptide-Y-Y1-receptor-expressing neurons in the paraventricular hypothalamic nucleus of the mouse. Neuroendocrinology. 70:295-305.

20. Jain, M.R., Horvath, T.L., Kalra, P.S., and Kalra S.P. 2000. Evidence that NPY Y1 receptors are involved in stimulation of feeding by orexins (hypocretins) in sated rats. Regul. Pept. 87:19-24.

21. Chaffer, C.L., and Morris, M.J. 2002. The feeding response to melanin-concentrating hormone is attenuated by antagonism of the NPY Y(1) receptor in the rat. Endocrinology. 143:191-197.

22. Sainsbury, A., Schwarzer, C., Couzens, M., and Herzog, H. 2002. Y2 receptor deletion attenuates the type 2 diabetic syndrome of ob/ob mice. Diabetes. 51:3420-3427.
23. Kushi, A., et al. 1998. Obesity and mild hyperinsulinemia found in neuropeptide Y-Y1 receptordeficient mice. Proc. Natl. Acad. Sci. U. S. A 95:15659-15664.

24. Barsh, G.S., and Schwartz, M.W. 2002. Genetic approaches to studying energy balance: percep tion and integration. Nat. Rev. Genet. 3:589-600.

25. Kalra, S.P., et al. 1999. Interacting appetite regulating pathways in the hypothalamic regulation of body weight. Endocr. Rev. 20:67-100.

26. Cowley, M.A., et al. 2001. Leptin activates anorexigenic POMC neurons through a neural network in arcuate nucleus. Nature. 411:480-484.

27. Cowley, M.A., et al. 2003. The distribution and mechanism of action of ghrelin in the CNS demonstrates a novel hypothalamic circuit regulating energy homeostasis. Neuron. 37:649-661

\title{
Renal fibrosis: not just PAI-1 in the sky
}

\author{
Agnes B. Fogo
}

Department of Pathology, Vanderbilt University Medical Center, Nashville, Tennessee, USA

A delicate balance exists between ECM synthesis and degradation such that interruption of the corresponding pathways results in increased plasminogen activator inhibitor-1 (PAI-1), pathological matrix accumulation, and glomerulosclerosis. A new study (see the related article beginning on page 379) demonstrates that therapy with a mutant PAI-1 increases matrix turnover and reduces glomerulosclerosis by competing with endogenous PAI-1, suggesting therapeutic utility in the treatment of fibrotic renal disease.

J. Clin. Invest. 112:326-328 (2003). doi:10.1172/JCI200319375.

Progressive fibrosis leads to endorgan failure in multiple organs, including heart, lung, liver, and kidney. The process of substitution of functional parenchymal cells by fibrotic tissue has been intensively investigated. Past progress over the last few decades has resulted in remarkable gains in slowing the progression of chronic kidney disease. The major emphasis has been on control of blood pressure, both systemi-

Address correspondence to: Agnes B. Fogo, MCN C3310, Department of Pathology,

Vanderbilt University Medical Center,

Nashville, Tennessee 37232, USA.

Phone: (615) 322-3114; Fax: (615) 343-7023;

E-mail: agnes.fogo@vanderbilt.edu.

Conflict of interest: The author has declared that no conflict of interest exists.

Nonstandard abbreviations used:

plasminogen activator inhibitor-1 (PAI-1);

tissue-type plasminogen activator (t-PA);

urokinase-type plasminogen activator

(u-PA); unilateral ureteral obstruction

(UUO); angiotensin type 1 (AT1);

epithelial-mesenchymal transition (EMT) cally and within the glomerulus. The efficacy of angiotensin I-converting enzyme inhibitors was linked to control of glomerular pressure beyond systemic pressure effects mediated by dilation of the efferent arterioles of the glomerulus (1). However, normalization of glomerular pressure did not completely halt progression, nor could it regress existing fibrosis. Numerous additional factors thus were implicated in progressive renal injury, including modulation of ECM and parenchymal and infiltrating cell interactions. In addition to angiotensin, TGF- $\beta$ has been recognized as a key mediator of renal fibrogenesis. Interestingly, both angiotensin and TGF- $\beta$ induce plasminogen activator inhibitor-1 (PAI-1) (2).

\section{PAl-1 and fibrosis mechanisms}

How might PAI-1 affect fibrosis? PAI-1 is the major inhibitor of tissuetype plasminogen activator ( $t-P A)$ and urokinase-type plasminogen activator (u-PA), which activate plasminogen to yield plasmin, which in turn degrades fibrin. PAI-1 not only inhibits fibrinolysis but also has complex interactions with matrix, promoting net proteolysis (3). t-PA, u-PA, and plasmin can degrade a wide range of ECM proteins; they also activate latent MMPs, in particular MMP-1 and MMP-3, and indirectly activate MMP-2. Models of glomerular sclerosis and/or interstitial fibrosis have been developed in both rats and mice to further examine these mechanisms. Noble and colleagues previously demonstrated that direct manipulation of the plasmin/plasminogen activator system with recombinant t-PA treatment decreased glomerular matrix accumulation in the anti-Thy1 model of glomerular matrix expansion (4). This model does not result in progressive renal damage and shows no attendant interstitial fibrosis but allows in vivo determination of mechanisms of glomerular matrix accumulation. Interstitial fibrosis can be induced in vivo in either rats or mice by unilateral ureteral obstruction (UUO). In contrast to the anti-Thy1 model, UUO does not result in significant glomerular lesions but demonstrates early macrophage infiltration and robust interstitial fibrosis, along with tubular injury. The unobstructed contralateral kidney serves as an ideal control. The UUO model thus allows in-depth in vivo study of mechanisms of interstitial fibrosis, and interactions between epithelial and infiltrating cells. The use of diverse models of renal scarring thus allows 